\title{
Research on the urban management monitoring and command center
}

\author{
Cheng Yunli ${ }^{1}$, Xue Huili ${ }^{2}$ \\ ${ }^{1}$ Department of Electronic Information Engineering, Guangzhou Nanyang Polytechnic \\ Guangzhou, China \\ ${ }^{2}$ Department of Electronic Information Engineering, Guangzhou Nanyang Polytechnic \\ Guangzhou, China
}

Keywords: Urban Management; Video Monitoring; Mobile Command

\begin{abstract}
In order to improve the level and efficiency of urban management in Heze, Shandong province, office of Heze urban management committee and the city construction information center want to build an urban management command center, to enhance the comprehensive law enforcement efficiency and the level of urban management through constructing the project of video monitoring and command system, as well as achieve the goal of establishing the "mobile command system and electronic monitoring system" by full use of the police, traffic police, traffic and video monitoring system.
\end{abstract}

\section{Introduction}

Digitization and intellectualization is the precondition of networking, while, networking is the basis of system integration. At present, system integration is growing rapidly, which will provide a more wide range of application fields for video monitoring system of urban management. The development of new technology, especially the technology of productization, can greatly reduce the technical risk of constructing an urban management command center.

For the purpose of improving the level and efficiency of urban management, the construction of an urban management command center for monitoring events, dispatching resources and dealing with urban management events rapidly and intensively, can improve the participation degree of social interaction in Heze, as well as provide technical support for improving the efficiency of comprehensive law enforcement, urban management level and service level.

\section{Main Research Content and Technical Route}

\section{Main Research Content.}

According to the actual situations, the video monitoring system of urban management covering Heze should be established. The specific requirement is to construct a video monitoring system of public security and comprehensive urban management, which basically covers the main city entrances and exits, key sites, main streets, key departments, people gathering places, public transport system, public places, communities and residential areas of Heze. In order to achieve the purpose of basically covering the whole city, the existing 90 thousand cameras and the related monitoring systems should be integrated and used, 160 thousand public security and urban management monitoring points should be new constructed. A total of 250 thousand cameras need to be built and transformed.

250 thousand cameras are not isolated islands, in order to manage so many cameras effectively, the video monitoring network covering the whole city need to be built. That is to build the whole city's video monitoring system with a network of three horizontals and two verticals. Which includes the public security video system with three level monitoring network of city bureau, branch office and local police station, as well as urban video system with three level monitoring network of municipality, district and street. At the same time, information sharing and mutual communication between each monitoring nodes of the video monitoring system should be realized.

In addition to the construction of cameras and monitoring networks, to achieve the purpose of 
improving the level of public security defense and control in the whole City of Heze, the collection, monitoring, storage, retrieval and comprehensive utilization for video information of social security and urban comprehensive management also need to be realized. Integrating the application systems of public security alarming linkage in Heze, GIS application, natural person and juridical person database etc., a monitoring, command and management system about urban management integrated videos is formed, which should focus on fighting, prevention and control for security of the whole society and take the urban management, emergency command, traffic management, safety production, environmental protection and water resources three proofing into account.

The overall structure of urban management video monitoring and command system in Heze is a structure of "five horizontals and two verticals". See Figure 1.

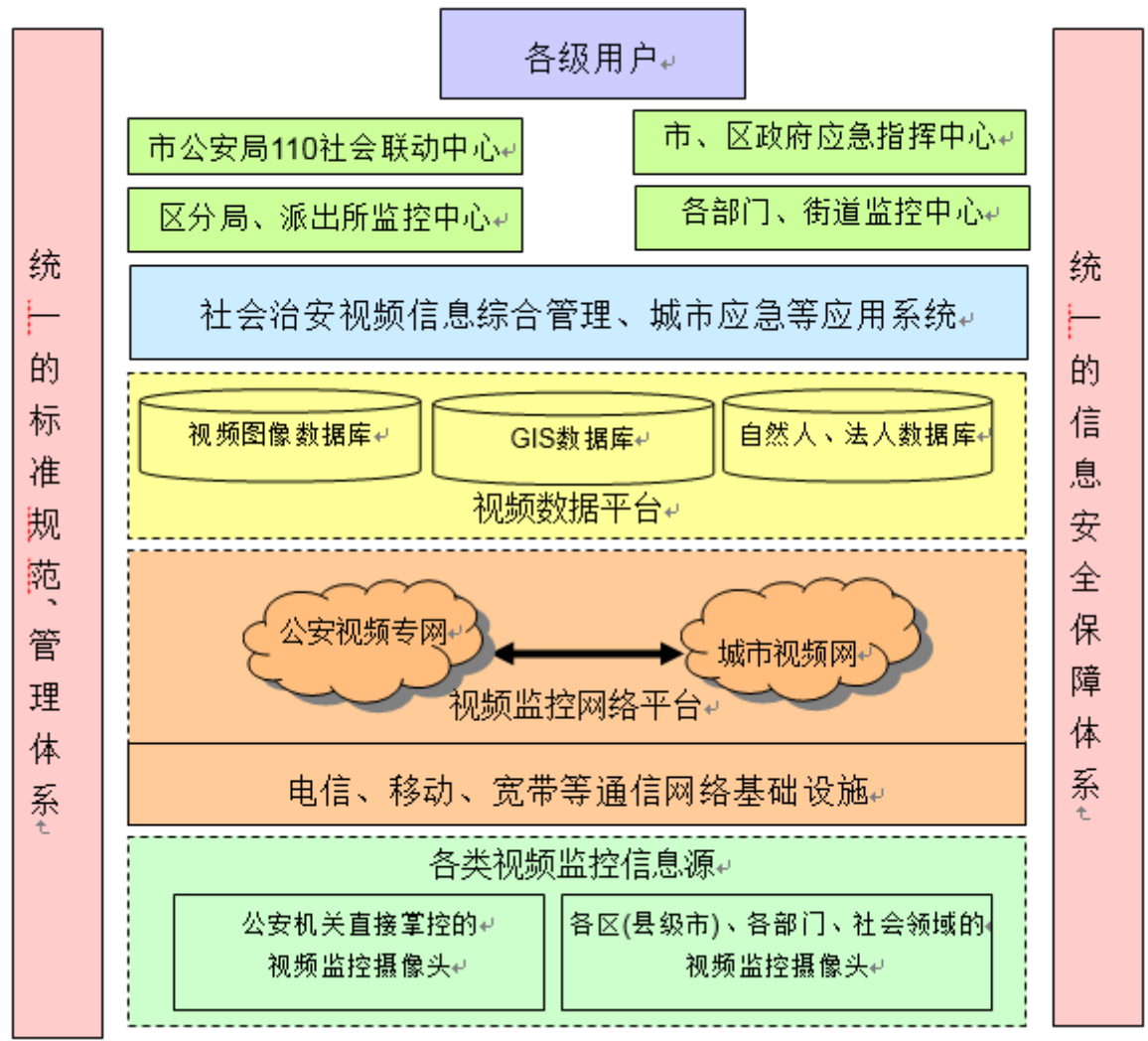

Figure 1. Overall structure of urban management video monitoring and command system in Heze

\section{Research Ideas.}

1) Based on urban management monitoring and command 2) Multi-department collaboration 3) Learning from the successful resource information 4) to achieve construction targets segmentally 5) by use of mature advanced technology 6) attaching equal importance to both management and information

For the purpose of improving the level and efficiency of urban management, the construction of an urban management command center for monitoring events, dispatching resources and dealing with urban management events rapidly and intensively, can improve the participation degree of social interaction in Heze, as well as provide technical support for improving the efficiency of comprehensive law enforcement, urban management level and service level.

\section{The Related Key Technologies.}

Microsoft .NET architecture is applied in this system. Microsoft .NET supports open standards, such as SOAP, Web Services Description Language(WSDL),Universal Description Discovery and Integration (UDDI) as well as XML. Microsoft provides a full range of support for the.NET framework, and possesses a variety of frame versions running on most Windows versions. This system is a multi-tier architecture, which adopts .NET framework. System Development Route and Method: .NET technical route, B/S application model. Operating Environment: .NET server. Development Tool: Visual Studio .NET.

Key Technologies: .NET development technology, data packets will be exchanged via 
SOCKECT mode and other systems.

\section{System Design}

\section{Network Structure.}

The network structure of video monitoring system for social security in Heze can be summarized as a structure of "Three horizontals and two verticals". "Three Horizontals" refers to the three levels of municipality, district (county-level city) and street (town), while "Two Verticals" refers to the private network of public security video (or net A for short) and the urban video network (or net B for short). The network topology of urban video private network and public security video private network is a four layer structure: core layer, gather high-layer, gather low-layer, access layer. Some of the corresponding network entities also can be divided into four level: primary monitoring center, secondary monitoring center, monitoring center of the third level, access point. As shown in figure 2.

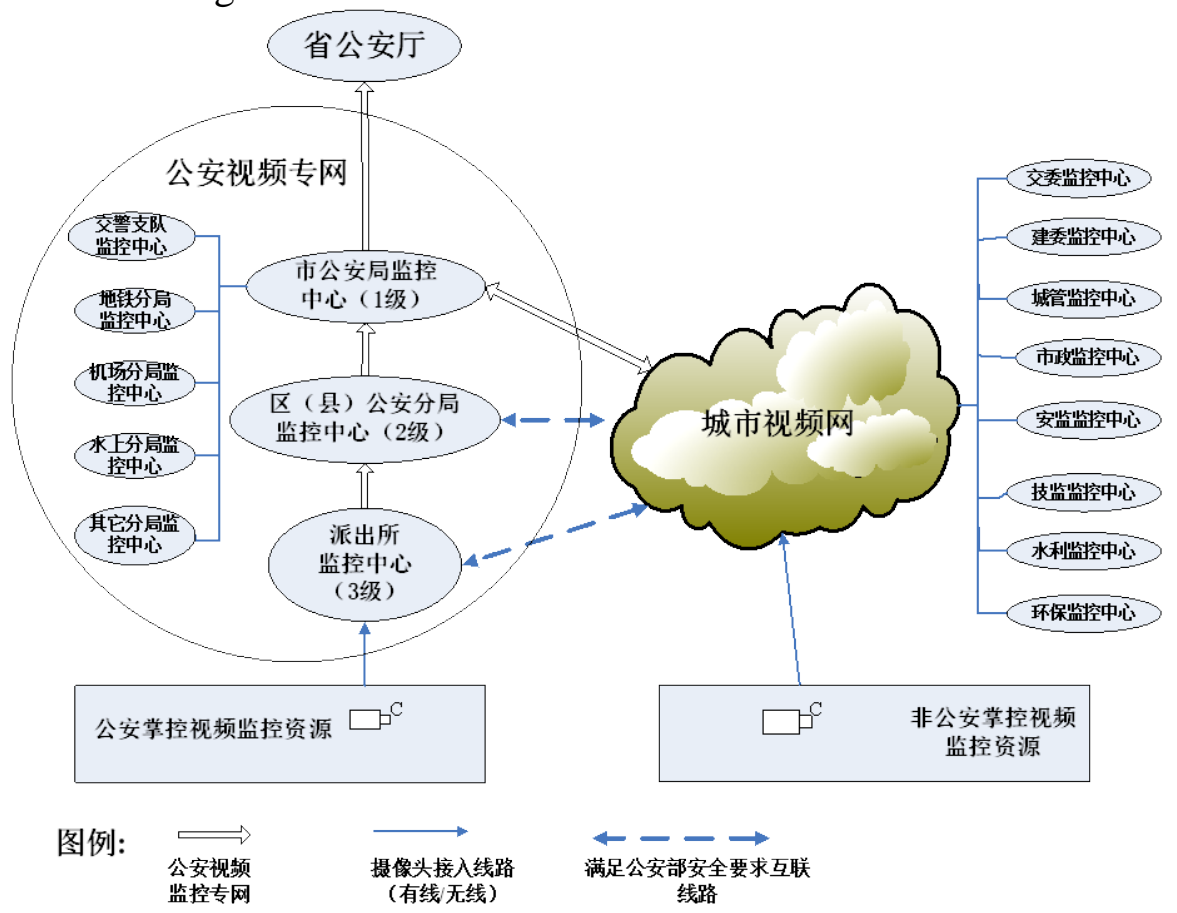

Figure 2. The overall network structure of video monitoring system for social security in Heze

Data from the video monitoring system for social security includes video data stream, data stream of control and management as well as application data stream. Where the video data stream includes digital video data and analog video data. The design of data layer is to describe the data format, transmission protocol and data exchange process.

For video data stream, a unified data communication format is required in data transmission. In all levels of monitoring centers, corresponding video decoding display and processing for different video data are carried out respectively. For controlling the data stream, the control commands with a unified format in all levels of monitoring centers is required to control different equipment in the leading end. While, the management of data stream demands to achieve the management for the whole network users' permissions and standing book.

For the GIS geographic information database system, the respective application of GIS geographic information system for the public security video private network and the urban video network is demanded, and which can be used for adjusting, controlling and managing the video monitoring resources in the whole network.

\section{Methods of Access to Social Resources.}

Social resources are the video monitoring system established by enterprises and institutions, social organizations and residential communities, etc., which is responsible for the access and management of video resources self-established by social units. Social resources adopts the 
following access methods, as shown in figure 3:

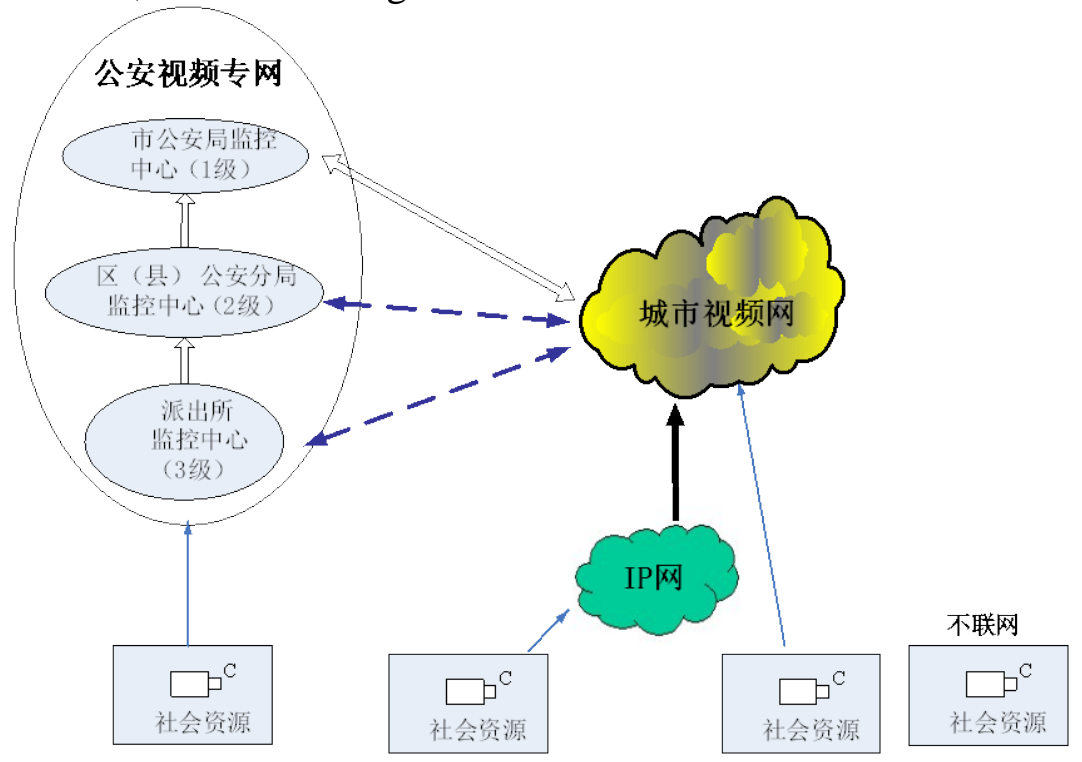

Figure 3. Schematic diagram of access to social resources

Through the establishment of dedicated line fiber or cable, cameras of important units access to the urban video network and the public security video private network. The access to public security network should meet the requirements of information security.

Design of Data Layer.

The data of Heze social security video monitoring system mainly includes the following categories:

1) Video data, including real-time image data, historical image data, audio data, etc.

2) Geographic data, including basic geographic information data, monitoring point layer data, etc.

3) System management data, including monitoring node data, equipment data, users' permissions data, etc.

4) Alarm data, including alarm point information, monitoring point information, alarm images, etc. Alarm data switches to horizontal applications via data interfaces, for the use of horizontal emergency command system.

5) The video data can be obtained from video collection for the monitoring points; The position of the monitoring points can be displayed directly on the electronic map by the relationship between geographic location and geographic data of the monitoring points; Through the establishment of the relationship between monitoring points and the system management data, it can more effectively manage the monitoring points and collect videos from the monitoring point.

\section{Implementation of System Core Functions}

The implementation scheme of core functions in this system will be introduced in the following, from the point of the implementation of public security video private network. The structure of public security video monitoring system in Heze is shown in Figure 4: 


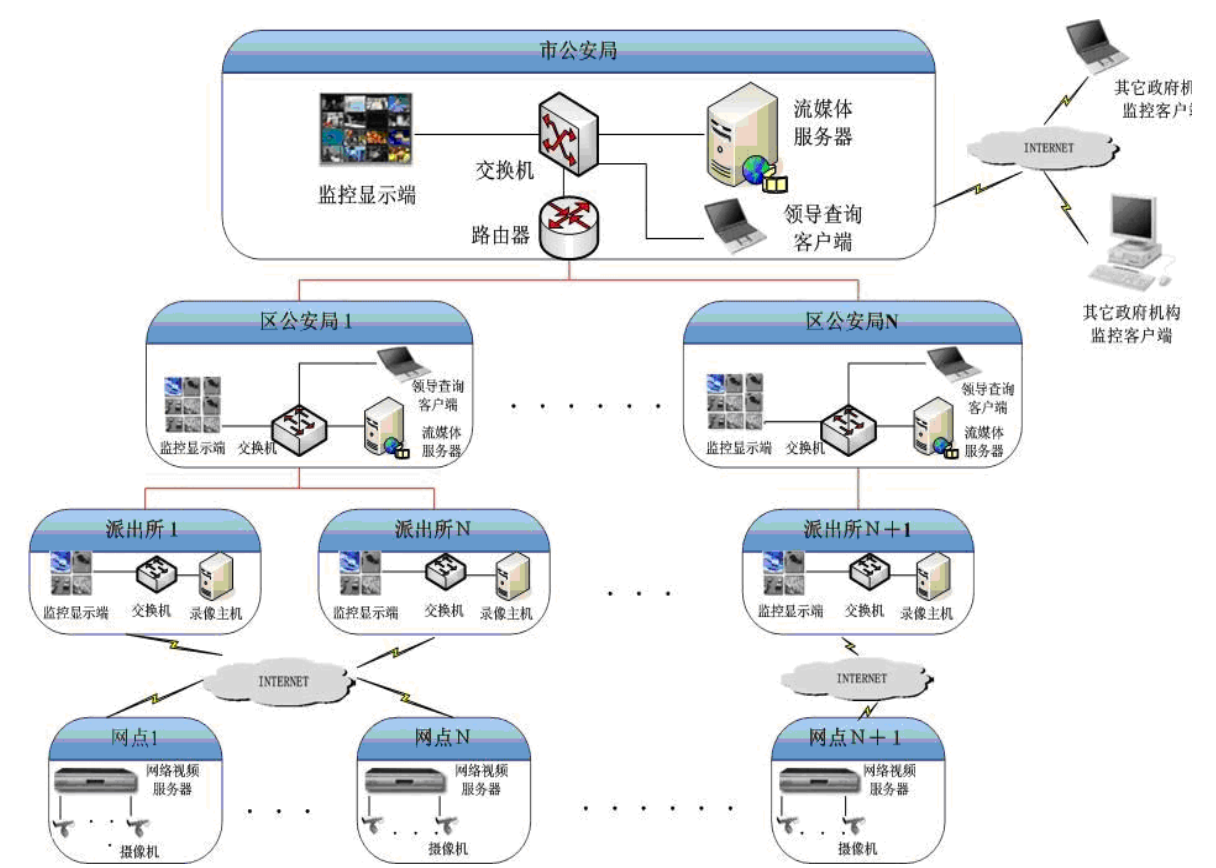

Figure 4. Structure chart of public security video monitoring system in Heze

1) Subsystem of monitoring leading end

The subsystems of monitoring leading end refers to that distribute in sensitive areas of public security appointed by the city's public security bureau, which includes each monitoring network in large-scale residential areas and commercial centers in flourishing regions, etc.

2) Transmission subsystem

Transmission subsystem includes digital transmission network and network port equipment. Transmission network in this system includes that between public security sub-bureau and municipal public security bureau, monitoring points in leading end and local police station, as well as local police station and public security sub-bureau.

3) Monitoring center and subsystem of monitoring client

Dividing from the area and permission, this system sets up a three level monitoring center-municipal public security bureau, public security sub-bureau and local police station, each monitoring center is responsible for the tasks of image monitoring within the area.

\section{Conclusion}

The video monitoring system of digital urban management is a new model of information society and urban management, it integrates the urban space into a visual electronic geographic map through the satellite navigation and positioning, wireless video and other technologies, comprehensively utilizing the extensive applications of computer information technology, GIS technology, network technology and image processing technology in urban management video monitoring system, So as to realize the development trend from passive urban management monitoring to active monitoring.

\section{Reference}

[1] Fuqiang Liu, Development and Application of Digital Video Monitoring System. Beijing: China Machine Press,2013

[2] Ling Zhang, Jingui Xu. Unlimited Business Opportunities of Video Monitoring System. Modern Economic Information(Academic Edition),2012

[3] Xiquan Gao, Yumei Ding, Yonghong Kuo. Digital Signal Processing_-Principle, Implementation and Application. Beijing: Publishing House of Electronics Industry,2012 
[4] Rui Yin, Guolong Wan. Digital Signal Processing. Beijing: Tsinghua University Press,2014

[5] Yonghua Liu, Fengqing Zhang, Jinling Zhou. Computer Networking and Maintenance Technology. Beijing: Tsinghua University Press,2013

[6] HESSLER S, WELZL M. An empirical study of the congestion re-sponse of RealPlayer, Windows MediaPlayer and Quicktime. IEEE Symposium on Computers and Communica-tions. 2015

[7] Xiaosheng Liu, Yuenan Wu, Shuang Zhou. An Introduction to the Engineering Technology of Intelligent Community System. Beijing: Publishing House of Electronics Industry,2011

[8] Wenbo Zheng. Technology of Control Network. Beijing: Tsinghua University Press,2011

[9] MASRY M, HEMAI S S. An analysis of subjective quality in lowbitrate video. IEEE International Conference on Image Pro-cessing. 2013

[10] Jinhui Liu. Network Design of Metro Video Monitoring System with Multi-Monitoring Center. Security and Safety Technology Magazine,2014

[11] Jie Tu. The Application of MPEG-4 in Digital Video Remote Monitoring System. Computer Applications and Software,2014

[12] Maggie Biggs. Power Designer speeds database development. InfoWorld, 2007

[13] Wenlun Cao. Research on Streaming Media of Image Compression and Wireless Network Traffic Video. Northwestern Polytechnical University,2012

[14] Wen Ji. Research on Key Technologies of Multimedia Communication based on Wireless Network. Northwestern Polytechnical University,2013

[15] Ming Li. Technology research and application case of monitoring and alarming network platform. China Security \& Protection, 2014 